\title{
Application of comprehensive advanced geological prediction technology in Da-puling tunnel
}

\author{
Hewen $\mathrm{Liu}^{1,2,3}$, Zhifa $\mathrm{Yu}^{1,2,3}$, and Hao Zhang ${ }^{1,2,3}$ \\ ${ }^{1}$ CCCC First Harbor Eng. Co., Ltd. \\ ${ }^{2}$ Tianjin Port Engineering Institute Co., Ltd. of CCCC First Harbor Eng. Co., Ltd. \\ ${ }^{3}$ Key Laboratory of Port Geotechnical Engineering of the Ministry of Communication Tianjin, China
}

\begin{abstract}
For the Da-puling tunnel of Puqing Expressway in Guangxi, the advanced geological prediction is carried out by combining TSP long-distance forecast method with short distance geological radar method. This paper describes the principle of seismic wave propagation in elastic medium, as well as the key points of data processing and analysis, some requirements that should be paid attention to the field test and scientific way of image interpretation put forward to improve the accuracy of prediction; When TSP is deployed, it should be sharp angle with potential joint surface. P-wave reacts surrounding rock properties, the shear wave is closely related to the transverse skeleton of medium. In data interpretation, it is necessary to focus on the analysis of the characteristics of P-wave and S-wave, weakening Poisson's ratio and Young's modulus. TSP and GPR can achieve the mutual complement and improve the detection accuracy.
\end{abstract}

\section{Introduction}

In recent years, with the improvement of industry laws and regulations, tunnel advance geological prediction has become an essential project in tunnel construction. Various geophysical methods based on the detection principles of electricity, magnetism and field strength are applied in tunnel advance prediction; it mainly includes geological logging, TSP, land sonar, ground penetrating radar, infrared water detection, transient electromagnetic method, high density electrical method, etc. Deng Youdong ${ }^{[1]}$ elaborated the application of $\mathrm{TSP}^{203}$ advanced geological prediction system in Wu Shaoling Super Long Tunnel, including data acquisition, data processing and interpretation results, and achieved good application effect. Wu Jun et al. ${ }^{22}$ cited several successful examples of geological radar applied in advance prediction of highway tunnel construction, and gave the radar waveform characteristics of several typical adverse geological bodies. On the basis of various geological prediction technologies, Li Shucai et al. [3] put forward the four-color early warning mechanism of tunnel, which has a significant effect on construction guidance. Due to different detection principles, various geophysical exploration methods have their own advantages and disadvantages in practical engineering application, corresponding to a certain scope of application, and a comprehensive prediction method combining multiple prediction methods is bound to become the development trend.

In this paper, we carried out the advanced forecast work of the Da-Puling tunnel in Puqing expressway, Guangxi, from the selection of forecast method to the determination of prediction process and data signal processing and interpretation, so as to achieve accurate prediction of strata information and scientifically guide the purpose of construction.

\section{Project overview}

Da-Puling tunne ${ }^{[4]}$ is located in TJ-3 contract section of Guangxi Puqing expressway. The design speed is $120 \mathrm{~km} / \mathrm{h}$. It is a separated two-way four lane tunnel with composite lining structure. The starting and ending mileage of the left tunnel is ZK24+270 ZK26+445, with a total length of $2175 \mathrm{~m}$. The starting and ending mileage of the right tunnel is YK24+280 YK26+435, with a total length of $2155 \mathrm{~m}$. The maximum excavation span of a single tunnel is $14.11 \mathrm{~m}$, and the maximum excavation height is $10.51 \mathrm{~m}$, the inner contour area of the standard section is $121 \mathrm{~m}^{2}$. The $\mathrm{V}$-grade surrounding rock is excavated by three-step reserved core soil method, and the designed reserved deformation is $12 \mathrm{~cm}$; Grade IV surrounding rock is excavated by two-step method, and the designed reserved deformation is $8 \mathrm{~cm}$.

The tunnel site is mainly Indosinian intrusive rock area, with undeveloped geological structure, but the weathered layer is thick, grade $\mathrm{V}$ surrounding rock accounts for about $12.6 \%$, class IV surrounding rock accounts for $18.3 \%$, and class III surrounding rock accounts for $69.1 \%$. The groundwater is mainly Quaternary loose void water and bedrock fissure water. 


\section{Advance prediction technology of tunnel}

\subsection{Geological logging}

Geological catalog is the drawing of face. Through the rock mass characteristics of the excavated section of the tunnel, it mainly refers to the surrounding rock characteristics of the face, including the stability, fragmentation, fracture development and water content of the rock mass. Based on the characteristics of rock stratum extension, the information of the rock layer in front of the tunnel is deduced. The accuracy of the method is high for fault and fracture zone. Generally, the influence range of fault or fracture zone is predicted by using basic trigonometric function according to the strike and inclination of the exposed fault or fracture zone.

\subsection{TSP (Tunnel seismic Prediction)}

TSP method uses the propagation of weak seismic wave in rock mass caused by artificial blasting, and receives the reflected wave through high sensitivity three component sensor. According to the propagation characteristics of the received S-wave and P-wave in the rock mass, the young's modulus, Poisson's ratio and the potential lithologic interface intersecting with the tunnel axis are obtained based on the empirical wave velocity and rock physical and mechanical parameter model, as shown in Figure 1. When the wave velocity increases, the density, strength and stability of the rock are better, the corresponding Young's simulation is larger, the Poisson's ratio is smaller, and vice versa.

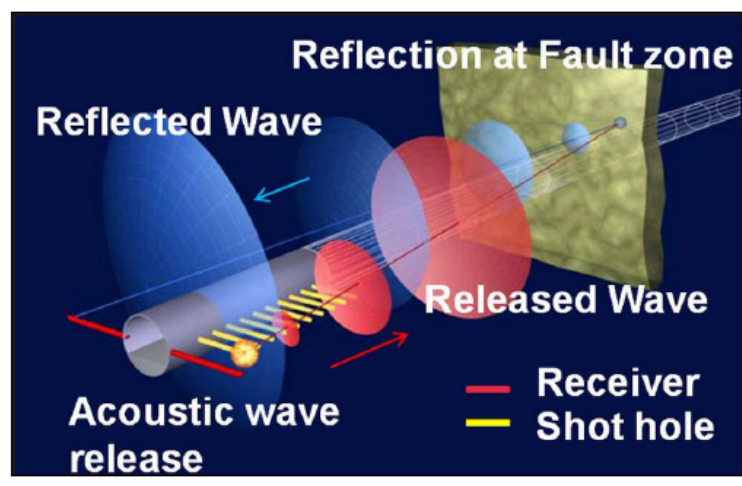

Fig. 1. Schematic diagram of TSP advanced geological prediction $^{[5]}$

\subsection{Ground penetrating radar}

The GPR transmits short pulse electromagnetic wave forward by antenna close to the face of the palm. After refraction and reflection of rock strata within $30 \mathrm{~m}$ in front, the echo is received on the face of the face. The spatial position of rock stratum and unfavorable geological body can be judged by analyzing the spectrum characteristics of reflection wave. In the process of electromagnetic wave propagation, reflection occurs at the interface of different media, the greater the material difference between the two sides of the interface, the more obvious the reflection. The results show that the amplitude, frequency, energy attenuation speed and the continuity of the same phase axis are different; In addition, electromagnetic wave is sensitive to water rich area, and the wave features of water phase reverse, low frequency, strong emission at the interface and rapid energy attenuation are found. Generally, the line $1^{[6,7]}$ is arranged at the place $1.5 \mathrm{~m}$ away from the ground on the face of the face. The layout of the line can be flexibly adjusted according to the surrounding rock conditions on the site, such as "soil" type and "well" type line 2-5, as shown in Figure 2.

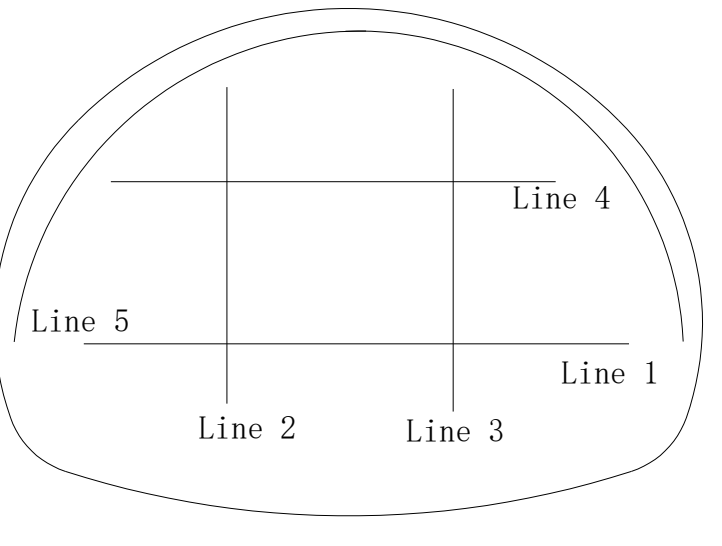

Fig. 2. The survey line layout of GPR

\section{Data acquisition and signal processing analysis}

In Da-Puling tunnel, TSP method and GPR method are used for advanced geological prediction; Because the TSP method needs 50 meters of working space, the geological radar method is used to forecast the entrance section; In addition, due to the low prediction accuracy of TSP method, GPR is used in both geological anomaly and potential unfavorable geological section. The combination of long-distance prediction and short-distance prediction, the combination of seismic wave method and electromagnetic wave method, learn from each other, improve the prediction accuracy, and provide scientific guidance for construction.

\subsection{TSP advanced forecast}

The new TSP is used in TSP ${ }^{303}$ plus, which has obvious advantages over $\mathrm{TSP}^{203}$. $\mathrm{TSP}^{303}$ plus needs to arrange seismic wave receiving sensors symmetrically on both sides of the tunnel to realize its three-dimensional detection purpose. Therefore, at least two sensors should be arranged in data acquisition, and the wave velocity of the medium should be determined by the iteration of the two sensors, it can more accurately determine the spatial location and distribution characteristics of the interface or adverse geological body.

In data acquisition, the side wall of the tunnel near the face should be selected in theory; 24 shot points are arranged, and the distance between shot holes is $1.5 \mathrm{~m}$. 
The final effective number of shot holes should not be less than 18. The amount of emulsion explosive should be $50 \mathrm{~g} \sim 300 \mathrm{~g}$. The better the surrounding rock is, the smaller the explosive is, and vice versa; The closer to the receiving sensor, the smaller the amount of charge used in the blast hole, and the farther the charge is, the more the amount of charge used. If it is impossible to determine on site, the charge can be loaded after the test.

The TSP prediction image is generated from the field data after several processing steps, such as band-pass filtering, first break picking, picking processing, gun energy equalization, Q-factor estimation, reflection wave extraction, P-wave(P) and S-wave(s) separation, velocity analysis, depth migration and reflection layer extraction. The TSP forecast image at the heading face mileage ZK24+576 of Da-Puling left line is shown in Figure 3.

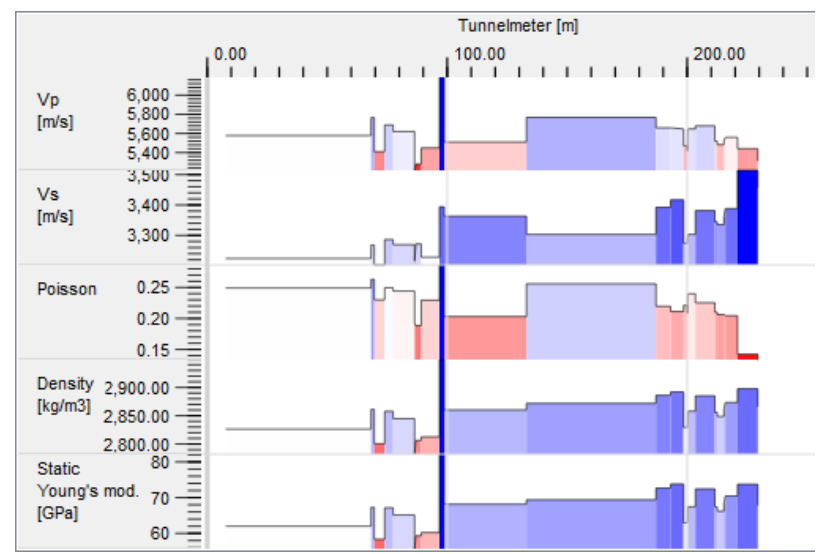

Fig.3. The 2D results chart of TSP signal

Figure 3 shows that the P-wave velocity of surrounding rock is $5200-5600 \mathrm{~m} / \mathrm{s}$ within $146 \mathrm{~m}$ in front of the tunnel face; The shear wave velocity is $3100 \sim$ $3500 \mathrm{~m} / \mathrm{s}$; The Poisson's ratio ranged from $0.13 \sim 0.24$; The density is $1.55-1.75 \mathrm{~g} / \mathrm{cm}^{3}$ and Young's modulus is 67-73 GPa.

The surrounding rock density of ZK24+576 ZK24+644 section is generally at a higher value, and the quality of surrounding rock is better. Compared with the surrounding rock in the face of the face, the property of surrounding rock is slightly worse. At the same time, the horizontal section and vertical section are alternately distributed in red and blue, and there are many joint fracture surfaces; The longitudinal wave velocity of ZK24+644 ZK24+694 increases, Poisson ratio decreases, and the horizontal section and vertical section are uniform, and the surrounding rock quality of this section is good; The longitudinal wave velocity of ZK24+694 ZK24+722 is slightly lower than that of the previous section, Poisson ratio increases and the integrity of surrounding rock decreases. However, the forecast distance of this section is more than $100 \mathrm{~m}$, and it is affected by many interference signals, so the reliability of the signal is reduced.

The reflectance image of prediction section is shown in Figure 4. The image shows that there are many reflection interfaces in ZK24+576 ZK24+644 section, the joint surfaces of surrounding rock are staggered, and the surrounding rock is broken. After that, the reflection surface of surrounding rock is significantly reduced, and the rock integrity is improved.

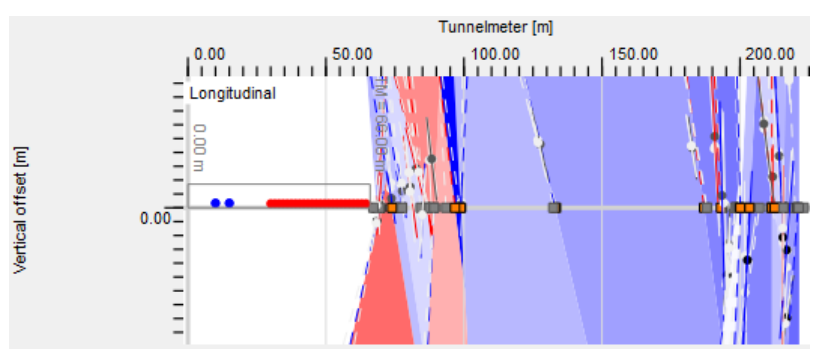

(a) Side view of reflector

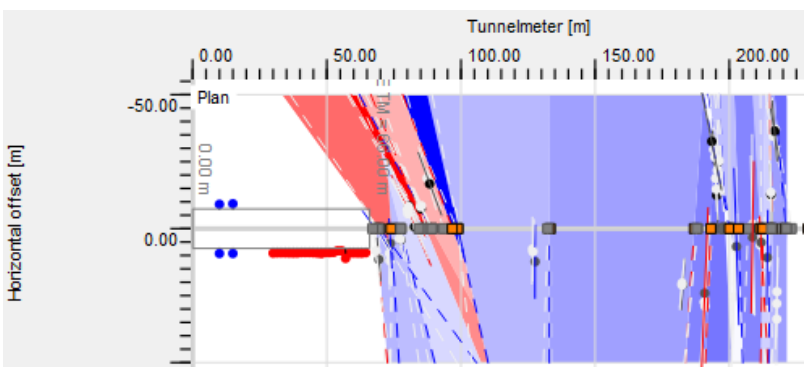

(b) Top view of reflector

Fig.4. Reflection layer image of TSP

$\mathrm{TSP}^{303}$ plus 3D slice is shown in Figure 5. The geological condition of any section of surrounding rock can be viewed through transverse slice and longitudinal slice, and the geological information in the cylinder with a diameter of $100 \mathrm{~m}$ can be displayed.

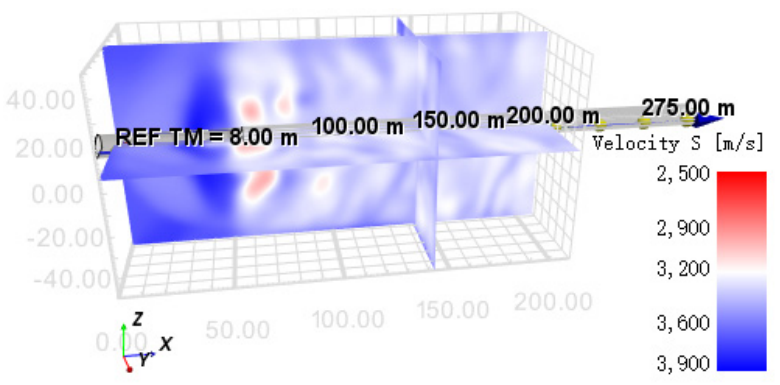

Fig. 5. The 3D imaging slice of TSP geological prediction

Based on the above information, advance geological prediction of tunnel surrounding rock can be made, as shown in Table 1. 
Table 1. Table of forecast results of TSP

\begin{tabular}{|c|c|c|c|c|}
\hline $\begin{array}{l}\text { Serial } \\
\text { number }\end{array}$ & mileage & Length/m & Forecast results & $\begin{array}{c}\text { Prediction of } \\
\text { surrounding rock } \\
\text { grade }\end{array}$ \\
\hline (1) & $\begin{array}{l}\mathrm{ZK} 24+576 \\
\mathrm{ZK} 24+644\end{array}$ & 68 & $\begin{array}{l}\text { It is inferred that the lithology of the surrounding rock of this } \\
\text { section is worse than that of the face, the rock is broken and } \\
\text { hard, the joint fissures are developed, the fissure water is } \\
\text { developed, and the overall stability of the surrounding rock is } \\
\text { poor. }\end{array}$ & $\begin{array}{l}\text { III (Strengthen } \\
\text { support) }\end{array}$ \\
\hline (2) & $\begin{array}{c}\mathrm{ZK} 24+644 \\
\sim \mathrm{ZK} 24+694\end{array}$ & 50 & $\begin{array}{l}\text { It is preliminarily judged that the surrounding rock of this } \\
\text { section is relatively hard rock, the rock mass is relatively } \\
\text { complete, the joints and fissures are weakly developed, and the } \\
\text { overall stability of the surrounding rock is general. }\end{array}$ & III \\
\hline (3) & $\begin{array}{l}\mathrm{ZK} 24+694 \\
\mathrm{ZK} 24+722\end{array}$ & 28 & $\begin{array}{l}\text { It is preliminarily judged that the surrounding rock of this } \\
\text { section is relatively hard rock, the rock mass is relatively } \\
\text { complete, the joint fissures are relatively developed, the fissure } \\
\text { water is weakly developed, and the overall stability of } \\
\text { surrounding rock is general. }\end{array}$ & III \\
\hline
\end{tabular}

\subsection{Super strong prediction of ground penetrating radar}

In order to further verify the prediction results of TSP in ZK24+694 ZK24+722 section, the geological radar advance geological prediction is carried out at ZK24+700 of heading face. The face of the tunnel is grayish white strongly to moderately weathered granite with hard texture and multiple closed fissures. The face of the tunnel is relatively wet. The scene photo is shown in Figure 6.

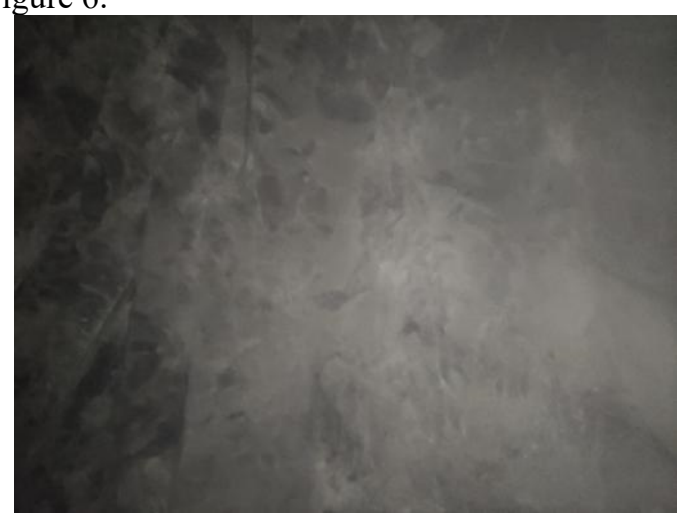

Fig.6. Face geolograph of tunnel

The ground penetrating radar (GPR) adopts $100 \mathrm{MHz}$ shielded antenna of laurel. The detection is carried out by point measurement. The time window is $600 \mathrm{~ns}$, the band-pass filter is $25 \sim 250 \mathrm{MHz}$, the number of sampling points is 1024 , and the static superposition is 5 times.

The detection image is shown in Figure 7. The image shows that the effective signals within $30 \mathrm{~m}$ range of this detection are strong, which indicates that the detection of surrounding rock has good integrity. The rock layer within $3 \mathrm{M}$ depth is affected by excavation and blasting loosening, and the interference of construction trolley. The radar wave has strong reflection, the same phase axis is broken, the image is distorted, and the reference is poor. The reflection energy of ZK24+703 ZK24+730 section is relatively uniform, and several groups of strong reflection in-phase axes are occasionally seen, which indicates that there are some joints and fissures in the surrounding rock of this section. Meanwhile, the frequency of the whole waveform is at a medium high level, and the water content is small, which is basically consistent with the fracture mileage predicted by TSP, and further confirms the properties of surrounding rock.

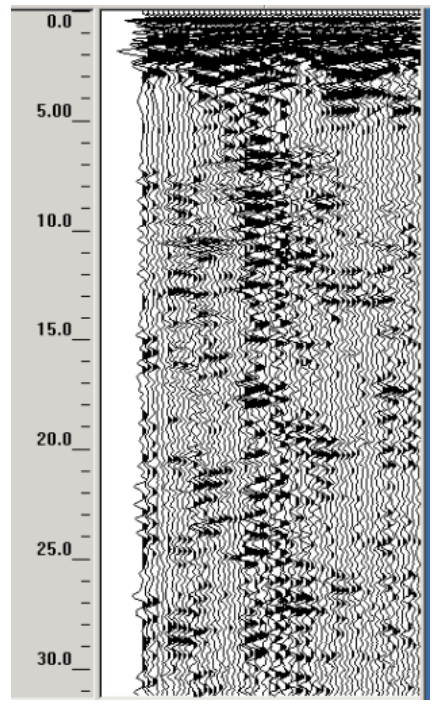

Fig.7. The detection section of GPR

\section{Conclusion}

(1) For Da-Puling tunnel, the comprehensive advanced prediction method combining face geological logging, TSP and geological radar method is adopted. The application effect in practical engineering prediction is good, which can provide reference value for tunnel advanced prediction engineering.

(2) Geophysical prospecting has multiple solutions, and usually a single prediction technology is easy to cause misjudgment. The combination of TSP and GPR can achieve the complementary prediction accuracy of long-range prediction and short-range prediction, and make full use of their distinguishing advantages for different geological bodies, which can well overcome this problem and detect the spatial position and geometric shape of bad geological bodies, It can 
effectively improve the prediction accuracy and ensure the construction safety.

(3) For advanced geological prediction, the quality of original signal collected on site is the key. For TSP detection, the bottom of the receiving hole should be vibrated repeatedly to ensure that the anchorage agent is filled tightly, and the top of $\operatorname{TSP}^{303}$ should be sealed with piston to ensure that the three-component receiver receives weak blasting head wave; Before blasting, the explosive is inserted into the bottom of the blasting hole, and the blasting hole is sealed with on-site tap water. The effect of waveform gain before radar detection has a great influence on the waveform quality. The gain parameters should be adjusted repeatedly to make the waveform scientific and reasonable. At the same time, the antenna should be close to the surrounding rock to ensure complete coupling before data collection. The forecast work should also coordinate the construction process to reduce the influence of external interference sources.

\section{References}

1. Deng Youdong. Application of geologic forecast in wu shaoling long tunnel. Chinese Journal of Rock Mechanics and Engineering, S2:5140-5146 (2004)

2. Wu Jun, Mao Hai-he, Ying Song, et al. Application of ground probing radar to short-term geological forecast for tunnel construction. Rock and Soil Mechanics, S1: 154-157 (2003)

3. Li Shucai, Xue Yiguo, Zhang Qingsong, etal. Key technology study on comprehensive prediction and Early-warning of geological hazards during tunnel Construction in high-risk karst areas. Chinese Journal of Rock Mechanics and Engineering, 07:1297-1307 (2008)

4. CCCC Second Highway Survey, design and Research Institute Co., Ltd. two stage construction drawing design of Guangxi Pubei Bei-liu Expressway (tj-03 bid section). Wuhan: CCCC Second Highway Survey, design and Research Institute Co., Ltd.

5. $\mathrm{TSP}^{303}$ plus operation manual. Amberger, Switzerland.

6. Li Tianyi. Study on Application of Ultra-low Frequency Ground Penetrating Radar in Karst Exploration. Journal of Railway Engineering Society. 03:6-11 (2012)

7. Li Tianbin, Meng Lubo, Zhu Jin, et al. Comprehensive analysis method for advanced forecast of Geology in tunnels[J]. Chinese Journal of Rock Mechanics and Engineering.28(12):2429 -2436 (2009) 